\title{
Comparison of stochastic Volterra equations
}

\author{
GUILLERMO FERREYRA* and PADAMANBHAN SUNDAR** \\ Department of Mathematics, Louisiana State University, Baton Rouge LA 70803, USA. \\ E-mail:*ferreyra@math.lsu.edu; **sundar@math.lsu.edu
}

A pathwise comparison theorem for a class of one-dimensional stochastic Volterra equations driven by continuous semimartingales is proved under suitable conditions. The result is applied to equations appearing in applications.

Keywords: semimartingale; stochastic differential equation; stochastic Volterra equation

\section{Introduction}

Let $\left(\Omega, \mathscr{F},\left\{\mathscr{F}_{t}: t \geqslant 0\right\}, P\right)$ be a probability space with the filtration satisfying the usual hypotheses. For each $j=1, \ldots, n$, let $\left\{M_{j}(t)\right\}$ be a real-valued continuous local martingale adapted to $\left(\mathscr{F}_{t}\right)$, and $\left\{V_{j}(t)\right\}$ be a continuous $\left(\mathscr{F}_{t}\right)$-adapted process, each with paths of bounded variation on compacts. Let $X_{i}(t), i=1,2$, be the pathwise unique strong solutions of the one-dimensional stochastic differential equations (SDEs)

$$
X_{i}(t)=\xi_{i}+\sum_{j=1}^{n} \int_{0}^{t} \sigma_{j}\left(t, s, X_{i}(s)\right) \mathrm{d} M_{j}(s)+\sum_{j=1}^{n} \int_{0}^{t} b_{j}^{i}\left(t, s, X_{i}(s)\right) \mathrm{d} V_{j}(s) .
$$

Such equations are known as stochastic Volterra equations and have been studied by several authors (see, for example, Berger and Mizel 1980; Cochran et al. 1995; Kolodii 1983; Protter 1985). The existence and uniqueness of solutions of stochastic Volterra equations driven by right-continuous semimartingales have been established by Protter (1985). A comparison theorem for solutions of stochastic equations with Volterra-type drifts was proved by Tudor (1989). We prove a comparison theorem for stochastic equations (1.1) where the diffusion coefficient is also of the Volterra type, namely when $\sigma_{j}(t, s, x)$ assumes the form $H(t) \sigma_{j}(s, x)$. It is important to note that a comparison of solutions of stochastic Volterra equations with general Volterra-type 'diffusion' coefficients is not possible. A counterexample is given in Tudor (1989). Our result is motivated by typical Volterra models that arise in practice. The method of proof employed in this paper is the variation of parameters which is akin to that in Protter (1990) for classical SDEs. 


\section{The comparison theorem}

We consider the following class of one-dimensional Volterra equations:

$$
X_{i}(t)=\xi_{i}+\sum_{j=1}^{n} H(t) \int_{0}^{t} \sigma_{j}\left(s, X_{i}(s)\right) \mathrm{d} M_{j}(s)+\sum_{j=1}^{n} \int_{0}^{t} b_{j}^{i}\left(t, s, X_{i}(s)\right) \mathrm{d} V_{j}(s),
$$

for $i=1,2$. We assume that $\{H(t)\}$ is a continuous, adapted, positive, strictly decreasing process. The coefficients $\sigma_{j}: \Omega \times \mathbb{R}^{+} \times \mathbb{R} \rightarrow \mathbb{R}$ and $b_{j}^{i}: \Omega \times \mathbb{R}^{+} \times \mathbb{R}^{+} \times \mathbb{R} \rightarrow \mathbb{R}$ are assumed to be jointly continuous for each fixed $\omega \in \Omega$, and satisfy a global Lipschitz condition in the space variable: $\left|\sigma_{j}(\omega, s, y)-\sigma_{j}(\omega, s, x)\right| \leqslant L|y-x|$ and $\mid b_{j}^{i}(\omega, t, s, y)-$ $b_{j}^{i}(\omega, t, s, x)|\leqslant L| y-x \mid$. We will suppress the first argument in the coefficients in the rest of the paper. The coefficients $\sigma_{j}(s, x)$ and $b_{j}^{i}(t, s, x)$ are $\mathscr{F}_{s}$ measurable for each $s \geqslant 0$. Moreover, we assume that the drift coefficients $b_{j}^{i}$ are differentiable in the first variable and $\left|D_{1} b_{j}^{i}(t, s, y)-D_{1} b_{j}^{i}(t, s, x)\right| \leqslant L|y-x|$, where $D_{1}$ denotes this first derivative. Then we can divide (2.1) by $H(t)$, and, letting $Y_{i}(t)$ denote $X_{i}(t) / H(t)$, obtain the following equation for $Y_{i}(t)$ :

$$
Y_{i}(t)=\frac{\xi_{i}}{H(t)}+\sum_{j=1}^{n} \int_{0}^{t} \sigma_{j}\left(s, Y_{i}(s) H(s)\right) \mathrm{d} M_{j}(s)+\sum_{j=1}^{n} \int_{0}^{t} \frac{b_{j}^{i}\left(t, s, Y_{i}(s) H(s)\right)}{H(t)} \mathrm{d} V_{j}(s) .
$$

Using integration by parts in the third term on the right-hand side, we obtain

$$
\begin{aligned}
Y_{i}(t)= & \frac{\xi_{i}}{H(t)}+\sum_{j=1}^{n} \int_{0}^{t} \sigma_{j}\left(s, Y_{i}(s) H(s)\right) \mathrm{d} M_{j}(s)+\sum_{j=1}^{n} \int_{0}^{t} \frac{b_{j}^{i}\left(s, s, Y_{i}(s) H(s)\right)}{H(s)} \mathrm{d} V_{j}(s) \\
& +\sum_{j=1}^{n} \int_{0}^{t} \frac{1}{H(s)} \int_{0}^{s} D_{1} b_{j}^{i}\left(s, r, H(r) Y_{i}(r)\right) \mathrm{d} V_{j}(r) \mathrm{d} s \\
& -\int_{0}^{t} \frac{1}{H^{2}(s)} \sum_{j=1}^{n} \int_{0}^{s} b_{j}^{i}\left(s, r, H(r) Y_{i}(r)\right) \mathrm{d} V_{j}(r) \mathrm{d} H(s) .
\end{aligned}
$$

Using the Picard iteration and the Doob inequality, it is a routine matter to establish the existence of a strong solution and pathwise uniqueness of solutions to (2.3). Hence we obtain the existence and uniqueness of $X_{i}(t)$. In order to prove a comparison theorem, we need the following additional hypotheses on the coefficients.

Hypotheses $\boldsymbol{H}$. Let $b_{j}^{i}: \Omega \times \mathbb{R}^{+} \times \mathbb{R}^{+} \times \mathbb{R} \rightarrow \mathbb{R}$ be such that, for each $\omega \in \Omega$, $i$ and $j$,

1. $b_{j}^{2}(s, r, y) \geqslant b_{j}^{1}(s, r, x)$ for all $0 \leqslant r \leqslant s$ and $y \geqslant x$;

2. $D_{1} b_{j}^{2}(s, r, y) \geqslant D_{1} b_{j}^{1}(s, r, x)$ for all $0 \leqslant r \leqslant s$ and $y \geqslant x$, where $D_{1}$ denotes the first derivative in the first variable. 
Before we proceed further, the following lemma is needed and is therefore stated without proof. This is the 'variation of parameters' formula in the stochastic set-up. A proof of it can be found in Protter (1990, pp. 266-267).

Lemma 2.1. Let $\left\{M_{t}\right\}$ be a continuous semimartingale starting at 0 , and $\left\{A_{t}\right\}$ be an adapted continuous process with paths of finite variation. If

$$
X_{t}=A_{t}+\int_{0}^{t} X_{s} \mathrm{~d} M_{s}
$$

then $X_{t}=\mathscr{E}(M)_{t}\left\{A_{0}+\int_{0}^{t} \mathscr{E}(M)_{s}^{-1} \mathrm{~d} A_{s}\right\}$, where $\mathscr{E}(M)_{t}$ is the stochastic exponential of $M$ given by $\mathrm{e}^{M_{t}-1 / 2\langle M\rangle_{t}}$.

Theorem 2.2. Assume Hypotheses $H$. If $V_{j}$ are increasing adapted processes for each $j$, and $\xi_{1} \leqslant \xi_{2}$, then $P\left(X_{1}(t) \leqslant X_{2}(t) \forall t \in \mathbb{R}^{+}\right)=1$.

Proof. Let $Y_{i}(t)$ denote $X_{i}(t) / H(t)$ as before. Let $Z_{t}=Y_{2}(t)-Y_{1}(t)$. From (2.2), $Z_{t}$ solves the equation

$$
\begin{aligned}
Z_{t}= & \frac{\xi_{2}-\xi_{1}}{H(t)}+\sum_{j=1}^{n} \int_{0}^{t} Z_{s} \mathrm{~d} N_{j}(s) \\
& +\sum_{j=1}^{n} \int_{0}^{t} \frac{1}{H(t)}\left(b_{j}^{2}\left(t, s, Y_{2}(s) H(s)\right)-b_{j}^{1}\left(t, s, Y_{1}(s) H(s)\right)\right) \mathrm{d} V_{j}(s),
\end{aligned}
$$

where

$$
N_{j}(t)=\int_{0}^{t} \frac{\sigma_{j}\left(s, Y_{2}(s) H(s)\right)-\sigma_{j}\left(s, Y_{1}(s) H(s)\right)}{Z_{s}} I_{\left\{Z_{s} \neq 0\right\}} \mathrm{d} M_{j}(s)
$$

are local martingales by the Lipschitz hypothesis on $\sigma_{j}$. Let $N_{t}=\sum_{j=1}^{n} N_{j}(t)$. Let $\mathscr{E}(N)_{t}$ denote the stochastic exponential of the local martingale $N$. Using Lemma 2.1, we can write (2.4) as

$$
\begin{aligned}
Z_{t}= & \mathscr{E}(N)_{t}\left[\frac{\xi_{2}-\xi_{1}}{H_{0}}-\int_{0}^{t} \frac{\mathscr{E}(N)_{s}^{-1}}{H(s)^{2}}\left(\xi_{2}-\xi_{1}\right) \mathrm{d} H(s)\right. \\
& +\sum_{j=1}^{n} \int_{0}^{t} \frac{\mathscr{E}(N)_{s}^{-1}}{H(s)}\left(b_{j}^{2}\left(s, s, Y_{2}(s) H(s)\right)-b_{j}^{1}\left(s, s, Y_{1}(s) H(s)\right)\right) \mathrm{d} V_{j}(s) \\
& -\frac{1}{H(s)} \int_{0}^{s}\left(b_{j}^{2}\left(s, r, Y_{2}(r) H(r)\right)-b_{j}^{1}\left(s, r, Y_{1}(r) H(r)\right)\right) \mathrm{d} V_{j}(r) \mathrm{d} H(s) \\
& \left.\left.+\int_{0}^{s}\left(D_{1} b_{j}^{2}\left(s, r, Y_{2}(r) H(r)\right)-D_{1} b_{j}^{1}\left(s, r, Y_{1}(r) H(r)\right)\right) \mathrm{d} V_{j}(r) \mathrm{d} s\right)\right]
\end{aligned}
$$


Let $J_{s}:=Z_{s} / \mathscr{E}(N)_{s}$. For any $u \leqslant s$, we can write

$$
\begin{aligned}
b_{j}^{2} & \left(s, r, Y_{2}(r) H(r)\right)-b_{j}^{1}\left(s, r, Y_{1}(r) H(r)\right) \\
\quad & =b_{j}^{2}\left(s, r, J_{r} \mathscr{E}(N)_{r} H(r)+Y_{1}(r) H(r)\right)-b_{j}^{1}\left(s, r, Y_{1}(r) H(r)\right) .
\end{aligned}
$$

The right-hand side of (2.6) will be denoted by $c_{j}\left(s, r, J_{r}\right)$. Let $D_{1} c_{j}$ denote the first derivative of $c_{j}$ with respect to the first variable. Equation (2.5) yields

$$
\begin{aligned}
J_{t}= & \frac{\xi_{2}-\xi_{1}}{H_{0}}+\int_{0}^{t} \frac{\mathscr{E}(N)_{s}^{-1}}{H(s)^{2}}\left(\xi_{2}-\xi_{1}\right) \mathrm{d}(-H(s)) \\
& +\sum_{j=1}^{n} \int_{0}^{t} \frac{\mathscr{E}(N)_{s}^{-1}}{H(s)}\left(c_{j}\left(s, s, J_{s}\right) \mathrm{d} V_{j}(s)+\frac{1}{H(s)} \int_{0}^{s} c_{j}\left(s, r, J_{r}\right) \mathrm{d} V_{j}(r) \mathrm{d}(-H(s))\right. \\
& \left.+\int_{0}^{s} D_{1} c_{j}\left(s, r, J_{r}\right) \mathrm{d} V_{j}(r) \mathrm{d} s\right) .
\end{aligned}
$$

We have thus written $J_{t}$ as the solution of a random integral equation with continuous increasing integrators. The following lemma finishes the proof.

Lemma 2.3. For each $i=1, \ldots, N$, assume that;

(i) $A_{i}$ are continuous increasing functions on $\mathbb{R}^{+}$.

(ii) $F_{i}: C[0, \infty) \rightarrow C[0, \infty)$ with $\left|F_{i}(u)_{t}-F_{i}(v)_{t}\right| \leqslant K_{i}(T) \sup _{0 \leqslant s \leqslant t}|u(s)-v(s)|$, for all $t \leqslant T$

(iii) for any given $t \geqslant 0, \quad u(s)=v(s) \forall 0 \leqslant s \leqslant t$ implies that $F_{i}(u)(s)=$ $F_{i}(v)(s) \forall 0 \leqslant s \leqslant t$, where $u, v \in C[0, \infty)$;

(iv) $u(t)=0 \Rightarrow F_{i}(u)_{t} \geqslant 0$.

Consider the integral equation

$$
U(t)=\xi+\sum_{i=1}^{N} \int_{0}^{t} F_{i}(U)_{s} \mathrm{~d} A_{i}(s),
$$

where $\xi \geqslant 0$. Then $U(t) \geqslant 0$ for all $t$.

Proof. Let $U_{\varepsilon}$ be the solution of $U_{\varepsilon}(t)=\xi+\sum_{i=1}^{N} \int_{0}^{t} G_{i}\left(U_{\varepsilon}\right)_{s} \mathrm{~d} A_{i}(s)$, where $G_{i}(u)_{s}=$ $F_{i}(u)_{s}+\varepsilon$. Then let $\tau=\inf \left\{t: U_{\varepsilon}(t)<0\right\}$. If $\tau<\infty$, then by continuity of $U_{\varepsilon}, U_{\varepsilon}(\tau)=0$. By condition (iv), $G_{i}\left(U_{\varepsilon}\right)_{\tau}>0 . G_{i}\left(U_{\varepsilon}\right) \in C[0, \infty)$ so that there exists a $\delta$ such that $G_{i}\left(U_{\varepsilon}\right)_{t}>0$ for all $t \in[\tau, \tau+\delta]$. Therefore $U_{\varepsilon}(t) \geqslant 0$ for all $t \in[\tau, \tau+\delta]$. This contradicts the finiteness of $\tau$. Therefore $\tau=\infty$. Thus $U_{\varepsilon}(t) \geqslant 0$ for all $t \geqslant 0$. Allowing $\varepsilon$ to tend to 0 , we obtain $U_{\varepsilon} \rightarrow U$ uniformly on $[0, T]$ for any $T \geqslant 0$. Therefore $U(t) \geqslant 0$ for all $t$.

Corollary 2.4. Let $V_{j}$ be increasing adapted processes for each $j$, and $\xi_{1} \leqslant \xi_{2}$. Let $b_{j}^{i}(t, s, x)=H(t) f_{j}^{i}(s, x) \quad$ for all $\quad 0 \leqslant s \leqslant t, \quad x \in \mathbb{R}, \quad i=1,2$ and $j=1, \ldots, n$. If 
$f_{j}^{1}(s, x) \leqslant f_{j}^{2}(s, x) \quad$ for $\quad$ all $\quad s \geqslant 0, \quad x \in \mathbb{R}, \quad$ and $\quad j=1, \ldots, n, \quad$ then $\quad P\left(X_{1}(t) \leqslant\right.$ $\left.X_{2}(t) \forall t \in \mathbb{R}^{+}\right)=1$.

Proof. For the particular form of the drift coefficients, equation (2.2) becomes

$$
Y_{i}(t)=\frac{\xi_{i}}{H(t)}+\sum_{j=1}^{n} \int_{0}^{t} \sigma_{j}\left(s, Y_{i}(s) H(s)\right) \mathrm{d} M_{j}(s)+\sum_{j=1}^{n} \int_{0}^{t} f_{j}^{i}\left(s, Y_{i}(s) H(s)\right) \mathrm{d} V_{j}(s)
$$

This is an SDE driven by continuous processes. The proof of the above comparison theorem yields the desired conclusion.

Example. Consider the following linear Volterra equation which arises in applications (for details, see Berger and Mizel 1980, Example 6B, p. 321; Miller 1971, p. 67):

$$
X_{i}(t)=\mathrm{e}^{-a t} \int_{0}^{t} \mathrm{e}^{a s}\left\{E_{i}(s)-f(s) X_{i}(s)\right\} \mathrm{d} s-\mathrm{e}^{-a t} \int_{0}^{t} \mathrm{e}^{a s} c(s) X_{i}(s) \mathrm{d} W(s),
$$

where $i=1,2$. Here $a>0$ and $E_{i}, f$ and $c$ are continuous functions of $s$. The above corollary implies that if $E_{2}(t) \geqslant E_{1}(t)$ for all $t>0$, then $X_{2}(t) \geqslant X_{1}(t)$ for all $t>0$ almost surely.

The above conclusion can also be obtained by considering the explicit solution of a linear stochastic equation. However, if the coefficients are nonlinear in the above equations, explicit solutions are seldom found. In addition, if the exponent -at is replaced by an adapted, decreasing and continuous process $A(t)$, our results seem to be the best possible.

Thus far we have assumed the differentiability of the drift coefficient in the first variable which allowed us to write the Volterra equation as an SDE with functional coefficients. A true Volterra drift is considered in the following theorem.

Hypotheses $\boldsymbol{H}^{\prime}$. Let $b_{j}^{i}: \Omega \times \mathbb{R}^{+} \times \mathbb{R}^{+} \times \mathbb{R} \rightarrow \mathbb{R}$ be such that, for each $\omega \in \Omega$, $i$ and $j$,

1. $b_{j}^{2}(s, r, y) \geqslant b_{j}^{1}(s, r, x)$ for all $0 \leqslant r \leqslant s$ and $x \leqslant y$;

2. $b_{j}^{2}\left(s^{\prime}, r, y\right)-b_{j}^{1}\left(s^{\prime}, r, x\right) \geqslant b_{j}^{2}(s, r, y)-b_{j}^{1}(s, r, x)$ for all $0 \leqslant r \leqslant s \leqslant s^{\prime}$ and $x \leqslant y$.

Theorem 2.5. Assume Hypotheses $\mathrm{H}^{\prime}$. If $V_{j}$ are increasing adapted processes for each $j$, and $\xi_{1} \leqslant \xi_{2}$, then $P\left(X_{1}(t) \leqslant X_{2}(t) \forall t \in \mathbb{R}^{+}\right)=1$.

Proof. Let $\varepsilon>0$ be fixed. Define $\bar{b}_{j}^{i}(t, s, x)=(1 / \varepsilon) \int_{t}^{t+\varepsilon} b_{j}^{i}(u, s, x) \mathrm{d} u$. Then $\bar{b}_{j}^{i}$ and $D_{1} \bar{b}_{j}^{i}$ are Lipschitz continuous in the space variable $x$ by using the Lipschitz property of $b_{j}^{i}$. Therefore, by the discussion at the beginning of this section,

$$
\bar{X}_{i}(t)=\xi_{i}+\sum_{j=1}^{n} H(t) \int_{0}^{t} \sigma_{j}\left(s, \bar{X}_{i}(s)\right) \mathrm{d} M_{j}(s)+\sum_{j=1}^{n} \int_{0}^{t} \bar{b}_{j}^{i}\left(t, s, \bar{X}_{i}(s)\right) \mathrm{d} V_{j}(s)
$$

admits a pathwise unique strong solution for $i=1,2$. Hypotheses $\mathrm{H}^{\prime}$ enable us to apply Theorem 2.2 to the solutions $\bar{X}_{i}$ of $(2.10)$, so that $P\left(\bar{X}_{2}(t) \geqslant \bar{X}_{1}(t) \forall t \in \mathbb{R}^{+}\right)=1$. 
Note that $\bar{X}_{i}(t)$ depends on $\varepsilon$, and we write it as $\bar{X}_{i}^{\varepsilon}(t)$ from now on:

$$
\mathrm{E} \sup _{t \in[0, T]}\left(\bar{X}_{i}^{\varepsilon}(t)-\bar{X}_{i}^{\varepsilon^{\prime}}(t)\right)^{2} \leqslant 8 C K^{2} \sum_{j=1}^{n} \mathrm{E} \int_{0}^{T}\left(\bar{X}_{i}^{\varepsilon}(t)-\bar{X}_{i}^{\varepsilon^{\prime}}(t)\right)^{2} \mathrm{~d}\left\langle N_{j}\right\rangle(t)
$$

As $\varepsilon_{n}$ decreases to $0, \mathrm{E}\left(\sup _{t \in[0, T]}\left|\bar{X}_{i}(t)-X_{i}(t)\right|\right)^{2}$ converges to 0 for any fixed $T$. Therefore, there is a subsequence of $\left\{\varepsilon_{n}\right\}$ along which the corresponding solutions $\left\{\bar{X}_{i}(t)\right\}$ converge to $\left\{X_{i}(t)\right\}$ uniformly on compacts almost surely. Therefore, $P\left(X_{1}(t) \leqslant X_{2}(t) \forall t \in \mathbb{R}^{+}\right)=1$.

\section{Acknowledgements}

The second author would like to thank the US Army Research Office for its support for this research through grant DAAH04-94-G-0249.

\section{References}

Berger, M. and Mizel, V. (1980) Volterra equations with Itô integrals. J. Integral Equations, 2, 187$245,319-337$.

Cochran, W.G., Lee, J.-S. and Potthoff, J. (1995) Stochastic Volterra equations with singular kernels, Stochastic Process. Appl., 56, 337-349.

Kolodii, A.M. (1983) On the existence of solutions of stochastic Volterra integral equations. Teor. Sluchajnykh Protsessov, 11, 51-57 (in Russian).

Miller, R.K. (1971) Nonlinear Volterra Integral Equations. Menlo Park, CA: W.A. Benjamin Inc.

Protter, P. (1985) Volterra equations driven by semimartingales. Ann. Probab., 13, 519-530.

Protter, P. (1990) Stochastic Integration and Differential Equations. Berlin: Springer-Verlag.

Tudor, C. (1989) A comparison theorem for stochastic equations with Volterra drifts. Ann. Probab., 17, $1541-1545$.

Received September 1998 and revised October 1999 